\title{
Tolerancing Tera-BREAD Using Ray-Tracing Simulations
}

\section{What is BREAD?}

BREAD is a dish-antenna style axion detection experiment currently in the design stage. It is a concept that allows broadband axion detection across a wide range of frequencies [1]. Axions interact with magnetic fields in the presence of conductors, emitting photons normal to the event surface in the process. BREAD is designed to fit into a solenoid magnet and focus the axion light to a focal spot. We currently plan on using an SNSPD, which has a dark count rate of around $1 /$ day

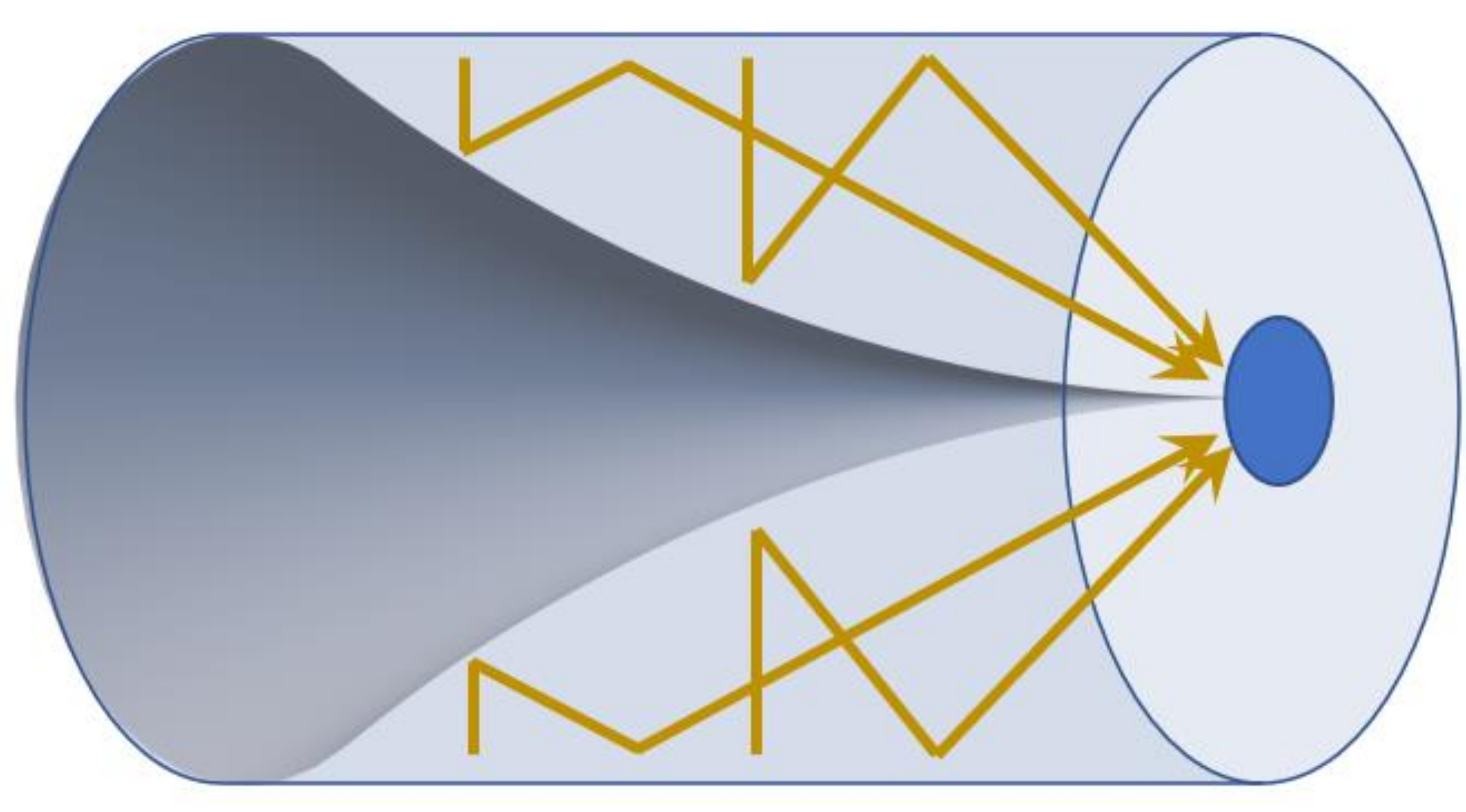

Concept art of BREAD. The instrument consists of a revolved parabolic reflector in a cylindrical container. BREAD is a dish antenna wrapped into a cylinder. We designed radius reflector. Fig. Courtesy Stefan Knirck (FNAL)

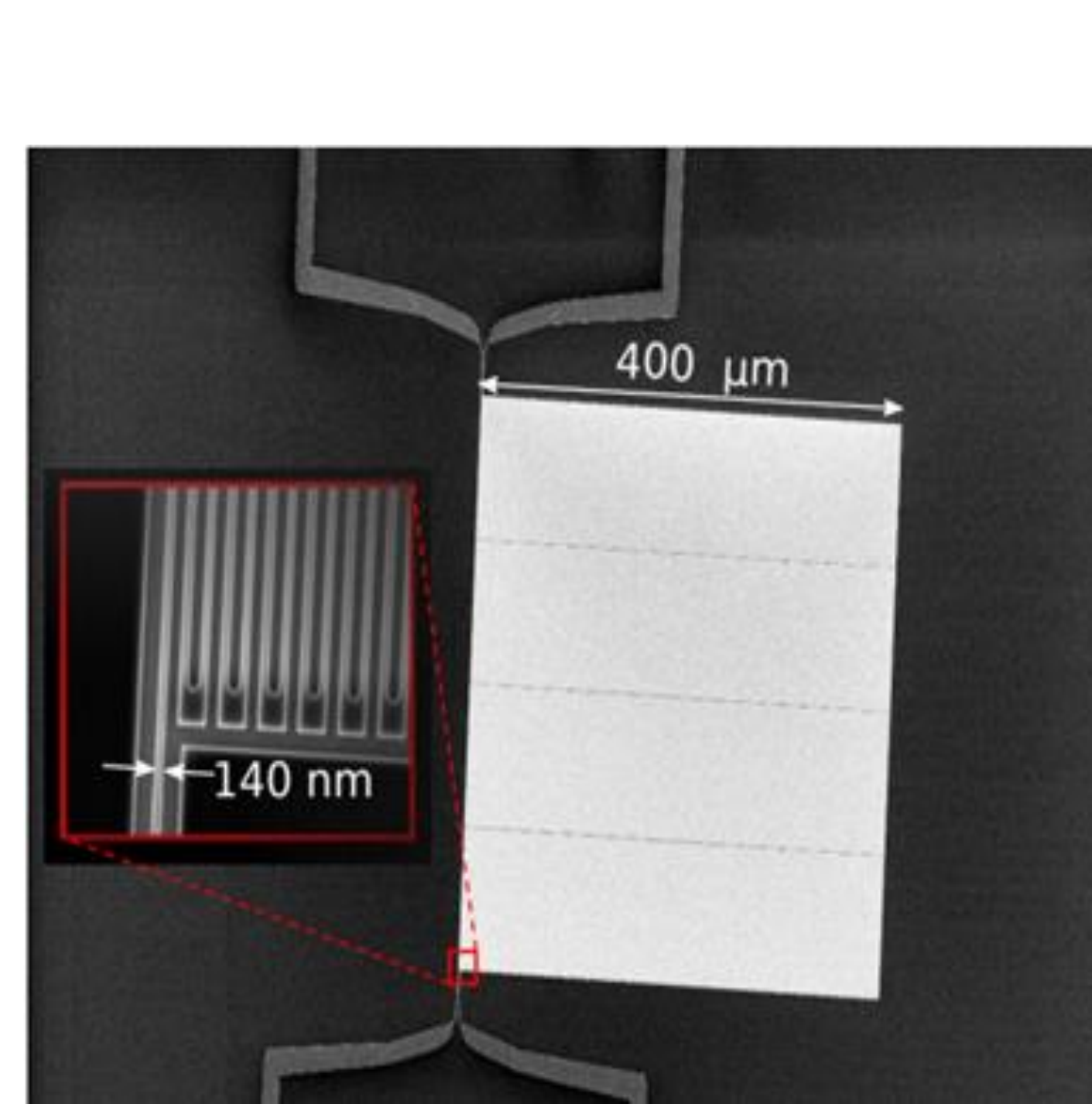

In order to deduce mechanical tolerances, we used FRED, a raytracing software package [3] Mechanical imperfections will defocus the beam, which must be kept in check because of the small size of SNSPDs, pictured on the left. This feature is the reason for this study. Our goal was to determine the

mage of a superconducting nanowire single photon detector
(SNSPD), which are typically $400 \mu m^{*} 400 \mu \mathrm{m}[2]$. mechanisms which broaden the [Hochberg et al., PRL 123 (2019)] spot size and in which ways they do so.

This manuscript has been authored by Fermi Research Alliance, LLC under Contract of High Energy Physics
Studies of Surface Ripples Using FRED

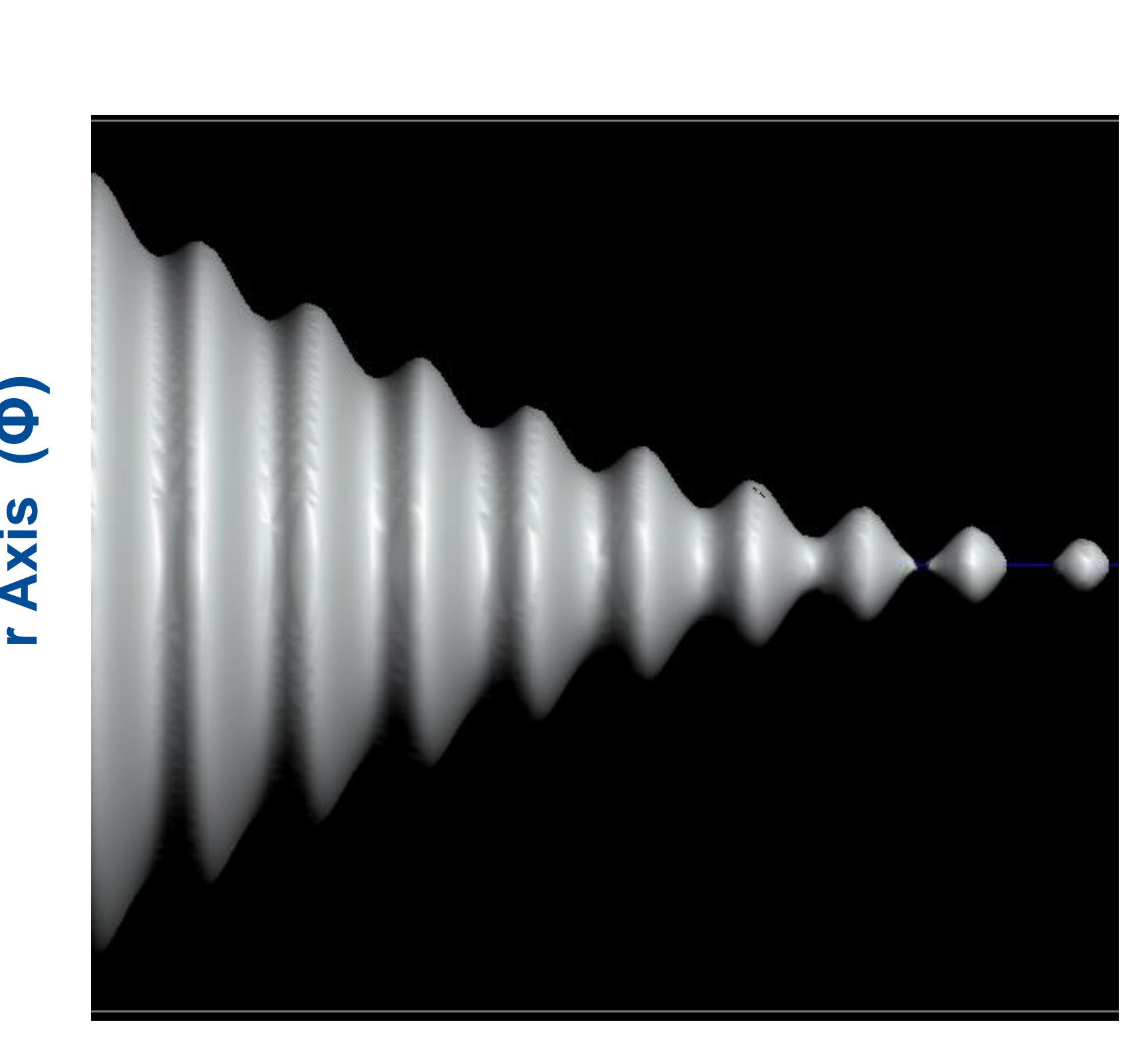

Z Axis Period ( ()

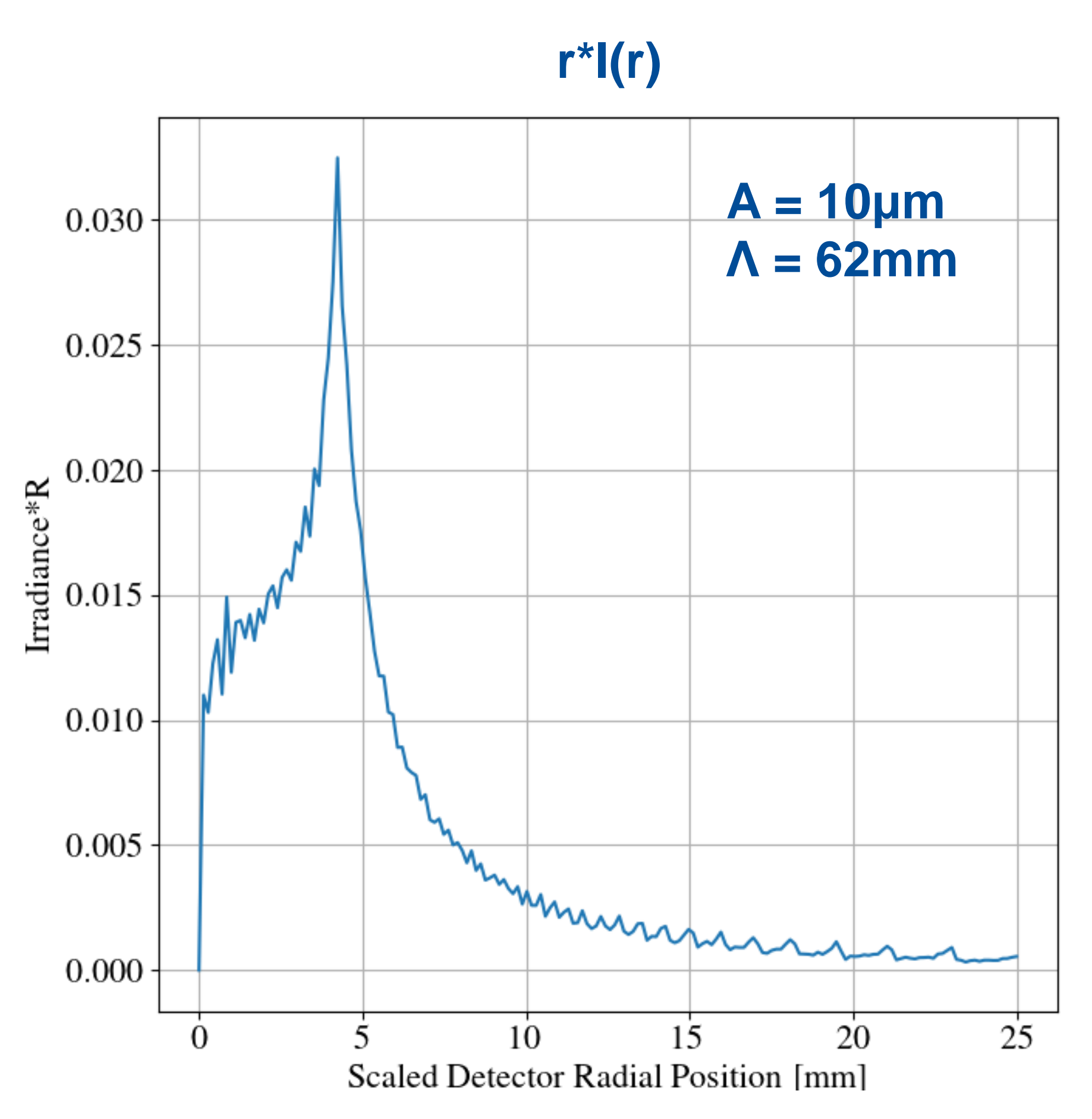

(Left) BREAD reflector with exaggerated ripple in Z direction. (Right) Reflector Z Ripple focal spot. As perturbations increase, these spots increase in size, but their features scale uniformly. Irradiance is multiplied by the radius to extract more information, such

Sinusoidal perturbations (ripples) cause a broadening of the focal spot. The width of the focal spot is determined by the product of the ripple angular frequency and amplitude. To first order, degeneracies in this parameter space yield the same spot size. This means that these two surface quality measures are coupled in terms of tolerances and must be treated accordingly.

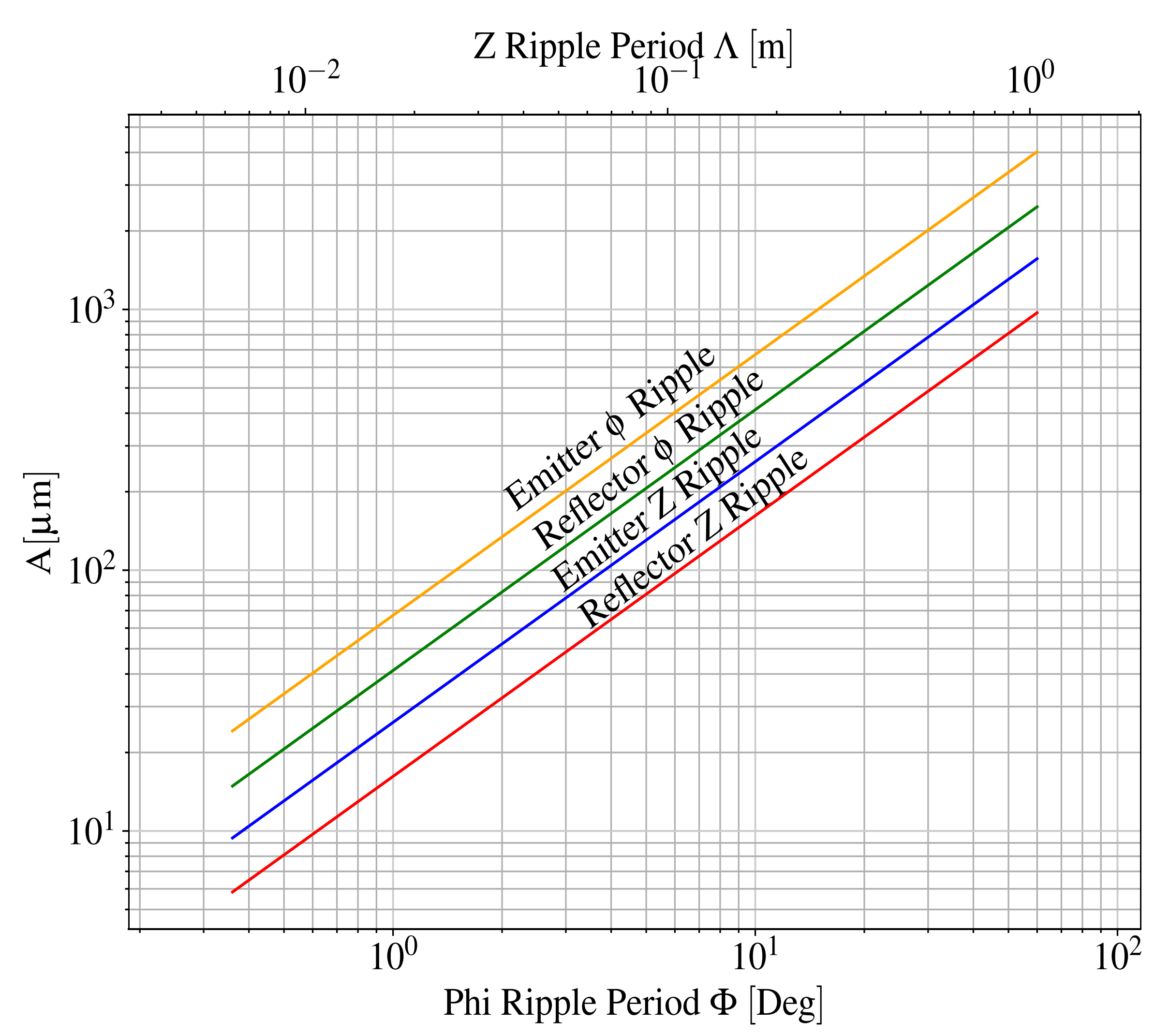

Each curve is a set of surface quality parameters that give a spot size of $0.5 \mathrm{~mm}$ in radius. Each curve is a different ripple, either in a different direction or on a different surface (e.g emitter or reflector)
We found that BREAD is significantly more sensitive to angular perturbations than positional ones. FRED defines surface positions and reflection angles separately, which enabled us to test the outcome of removing the position component of a perturbation. As such, the tolerances of the emitter spatial dimensions are much looser than any ripple. Surface ripples can cause significantly larger deflection angles than other perturbations and must be more tightly constrained as a result.

\begin{tabular}{|l|l|c|}
\hline Perturbation & Formula & Example Parameters \\
\hline $\begin{array}{l}\text { Z-ripple (Emitter) } \\
\text { Z-ripple } \\
\text { (Reflector) }\end{array}$ & $w=2100 * \frac{A}{\Lambda}$ & $\frac{w}{0.5 \mathrm{~mm}}=\frac{A}{20 \mu \mathrm{m}} * \frac{84 \mathrm{~mm}}{\Lambda}$ \\
\hline $\begin{array}{l}\text { D-Ripple } \\
\text { (Emitter) }\end{array}$ & $w=23400 * \frac{A}{\Lambda}$ & $\frac{w}{0.5 \mathrm{~mm}}=\frac{A}{20 \mu \mathrm{m}} * \frac{136 \mathrm{~mm}}{\Lambda}$ \\
\hline $\begin{array}{l}\text { D-Ripple } \\
\text { (Reflector) }\end{array}$ & $w=76000 * \frac{A}{\varphi}$ & $\frac{w}{0.5 \mathrm{~mm}}=\frac{A}{20 \mu \mathrm{m}} * \frac{0.94 \mathrm{Deg}}{\varphi}$ \\
\hline $\begin{array}{l}\text { Uniform Radial } \\
\text { Deviation }\end{array}$ & $w=1.98 *|\Delta R|$ & $\frac{w}{0.5 \mathrm{~mm}}=\frac{\mathrm{w}}{20 \mu \mathrm{m}} * \frac{3 \mathrm{Deg}}{\varphi}$ \\
\hline $\begin{array}{l}\text { Conical Radial } \\
\text { Deviation (Top) }\end{array}$ & $w=3.39 *|\Delta R|$ & $\frac{\mathrm{R}}{0.25 \mathrm{~mm}}$ \\
\hline $\begin{array}{l}\text { Conical Radial } \\
\text { Deviation } \\
\text { (Bottom) }\end{array}$ & $w=1.39 *|\Delta R|$ & $\frac{\mathrm{R}}{0.5 \mathrm{~mm}}=\frac{R}{0.15 \mathrm{~mm}}$ \\
\hline
\end{tabular}

\section{Conclusions}

In conclusion, we determined the tolerances to which the Tera-BREAD instrument must be fabricated. This includes the basic dimensions of each piece as well as constraining macroscopic ripples on each surface. Such ripples take the form of a sinusoidal perturbation, which showed that this design is more sensitive to deflection angles than to those in spatial coordinates.

\section{References}

[1] Horns et al, JCAP, Issue 04, article 016. arXiv:1212.2970

[2] Hochberg et al., PRL 123 (2019)

[3] FRED: https://photonengr.com/fred-software/ 